\title{
Increased Lysosomal Hydrolase Activity in Kidney and Brain from the Vitamin $B_{6}$-deficient Developing Rat
}

\author{
R. V. DIPAOLO, C. SPIELVOGEL, AND J. N. KANFER (35) \\ Eunice Kennedy Shriver Center for Research in Mental Retardation, W. E. Fernald State School, Waltham, and \\ Neurology Research, J. P. Kennedy, Jr., Memorial Laboratories, Massachusetts General Hospital, Boston, \\ Massachusetts, USA
}

\begin{abstract}
Extract
Between 7 and 9 postnatal days, the kidney, spleen, heart, and liver from vitamin $\mathbf{B}_{6}$-deficient young either plateaued and failed to gain weight or lost weight, whereas brain continued to gain weight but at a more reduced rate than normal. The level of cofactor in kidney of experimental animals was below that of controls at all time points studied. $\alpha$-L-Fucosidase activity was already moderately increased in deficient kidney at 3 postnatal days and remained elevated throughout the time course. $\beta$-D-galactosidase, acid phosphatase, and hexosaminidases $A$ and $B$ in vitamin $B_{6}$-deficient young all showed moderate increases in activities after 9 days, at one or more time points. In brain, $\alpha$-L-fucosidase activity was increased somewhat throughout the time course, but changes in $\beta$-D-glucuronidase, $\beta$-D-galactosidase, $\alpha$-D-mannosidase, acid phosphatase, and hexosaminidases $A$ and $B$ did not occur until after 7 postnatal days.
\end{abstract}

\section{Speculation}

When weights of kidney and brain were both normal, there was a marked elevation in $\alpha$-L-fucosidase activity in these organs suggesting that this increase in enzyme activity may be a specific effect of vitamin $B_{6}$ deficiency. Elevated $\alpha$-L-fucosidase activity in kidney may reflect alterations in fucose-containing glycosubstances of glomerular basement membranes.

Vitamin $B_{6}$ is essential to growth and survival of the rat. If dams are subjected to a vitamin $\mathrm{B}_{6}$-deficient diet during pregnancy or shortly after delivery of litters, pups fail to gain weight, become torpid, inactive, and develop tremors (3). Most young eventually appear moribund and $90 \%$ die between the 14th and 19th postnatal day (11). The content of vitamin $B_{6}$ is decreased in liver and brain. Symptoms and signs of failure coincide with elevated blood urea nitrogen and morphologic evidence of arrested renal development (3). The pace of differentiation of elements in the cortex is clearly delayed by the deficient state when compared with normal kidney of the same age. Unlike the normal, where mitoses are abundant in tubules, mitoses are rarely observed in any of the deprived animals. Retarded development of the splenic lymphoid follicles is also associated with vitamin $B_{6}$ deprivation and may reflect impaired cell division. Other organs are morphologically normal. The following study examines the activities of renal and cerebral lysosomal acid hydrolases in vitamin $\mathrm{B}_{6}$-deprived young.

\section{METHODS AND MATERIALS}

\section{ANIMALS}

Rats (Sprague-Dawley, CD strain), pregnant 1 day were purchased from Charles River Breeding Laboratories (27). They were divided in a random fashion upon arrival into control and deficient groups and housed separately in polycarbonate cages (28) containing Sani-Chips (29) as a bedding material. Litter size was reduced to eight animals within $24 \mathrm{hr}$ after birth.

\section{DIETS AND EXPERIMENTAL DESIGN}

The French (4) diet (30) was used to induce vitamin $B_{6}$ deficiency. The diet was offered to dams, ad libitum, starting 2 weeks after mating. Normal control dams were offered conventional laboratory chow (29).

Offspring were killed between 7 and $11 \mathrm{AM}$ at 3, 7, 9, 11, 14 , and 16 postnatal days. Animals were decapitated and blood was collected for urea nitrogen analysis (Diagnostest for urea nitrogen (31)). Liver, brain, kidney, spleen, and heart were excised immediately, weighed, and frozen. All measurements were made on individual samples. Control offspring of similar ages were also harvested and killed for comparative studies.

\section{LYSOSOMAL ACID HYDROLASE ACTIVITY}

Frozen whole kidneys were thawed and homogenized individually in $1 \mathrm{ml} 0.9 \%$ saline, and individual whole brains were homogenized in $2 \mathrm{ml}$ saline. Aliquots of the homogenates were removed for protein determinations (7) and the remaining portions refrozen. Before enzymic analysis the thawed homogenates were sonicated at an output setting of 4 with a sonifier cell disruptor (32), employing a semimicro tip, until the homogenates were uniformly dispersed. Aliquots were removed and assayed for several enzyme activities (Table 1) either directly or after being suitabiy diluted in saline. The assay conditions employed have been described previously $(8,9,17)$. The incubation mixtures were shaken at $37^{\circ}$ for $1 \mathrm{hr}$, followed by the addition of $0.7 \mathrm{ml} 2.75 \%$ trichloroacetic acid, $0.5 \mathrm{ml} 0.5 \mathrm{~N} \mathrm{NaOH}$, and $0.75 \mathrm{ml} 0.25 \mathrm{M}$ glycine buffer, $\mathrm{pH}$ 10.3. The 4-methylumbelliferone released was measured in an Amino-Bowman spectrofluorometer and the $p$-nitrophenol liberated was measured in a Beckman spectrophotometer. Each sample was assayed in duplicate at two levels of added protein.

Hexosaminidase $\mathrm{A}$ and $\mathrm{B}$ were assayed by the heating of aliquots of homogenates at $50^{\circ}$ for $2 \mathrm{hr}$ (18). Both total hexosaminidase and heat-stable enzyme, hexosaminidase $\mathrm{B}$, were assayed simultaneously. The difference between the two activities was taken to be hexosaminidase A activity.

\section{VITAMIN $B_{6}$ ANALYSIS}

The content of pyridoxal-5'-phosphate in kidney was quantitated employing the tyrosine apodecarboxylase method of analysis described previously $(11,15)$. The volume of perchloric acid used for extraction of the vitamin was based on the original wet weight of the kidney. Cofactor levels were measured in the same 
Table 1. Hydrolase activities quantitated in kidney and brain

\begin{tabular}{|c|c|c|c|}
\hline $\begin{array}{l}\text { Hydrolase }^{1} \\
\text { (EC. no.) }\end{array}$ & Substrate $^{2}$ & $\begin{array}{c}\text { Buffer }{ }^{3} \text { and other } \\
\text { additions }\end{array}$ & References \\
\hline$\alpha$-L-Fucosidase (3.2.1.5I) & $p$-Nitrophenyl- $\alpha$-L-fucoside & Citrate phosphate & 13,14 \\
\hline$\beta$-D-Glucuronidase (3.2.1.31) & 4-Methylumbelliferyl- $\beta$-D-glucuronide & Sodium acetate & 16 \\
\hline$\beta$-D-Galactosidase (3.2.1.23) & 4-Methylumbelliferyl- $\beta$ - $\mathrm{D}$-galactoside & Citrate phosphate & 21,22 \\
\hline$\alpha$-D-Mannosidase $(3.2 .1 .24)$ & 4-Methylumbelliferyl- $\alpha$-D-mannoside & Citrate phosphate & 6 \\
\hline Acid phosphatase (3.1.3.2) & 4-Methylumbelliferylphosphate & Sodium acetate & 23 \\
\hline$\beta$-D-N-Acetylhexosaminidase (3.2.1.52) & 4-Methylumbelliferyl- $\beta-\mathrm{D}-\mathrm{N}$-acetylglucosaminide & Citrate phosphate & 12 \\
\hline
\end{tabular}

${ }^{1}$ Enzyme numbers are according to the 1972 recommendations of the Commission on Biochemical Nomenclature.

${ }^{2}$ The final concentration of each substrate in the incubation mixture was $2.37 \mathrm{mM}$, excepting 4-methylumbelliferyl phosphate, which was at $0.356 \mathrm{mM}$.

${ }^{3}$ All enzyme activities were assayed at $\mathrm{pH} 4.5$ in the appropriate buffers.

${ }^{4}$ The incubation mixture also contained $0.19 \mathrm{M} \mathrm{NaCl}$ and $1 \mathrm{mg}$ bovine serum albumin in a final volume of $210 \mu 1$.

${ }^{5}$ Literature citations employing these artificial substrates.

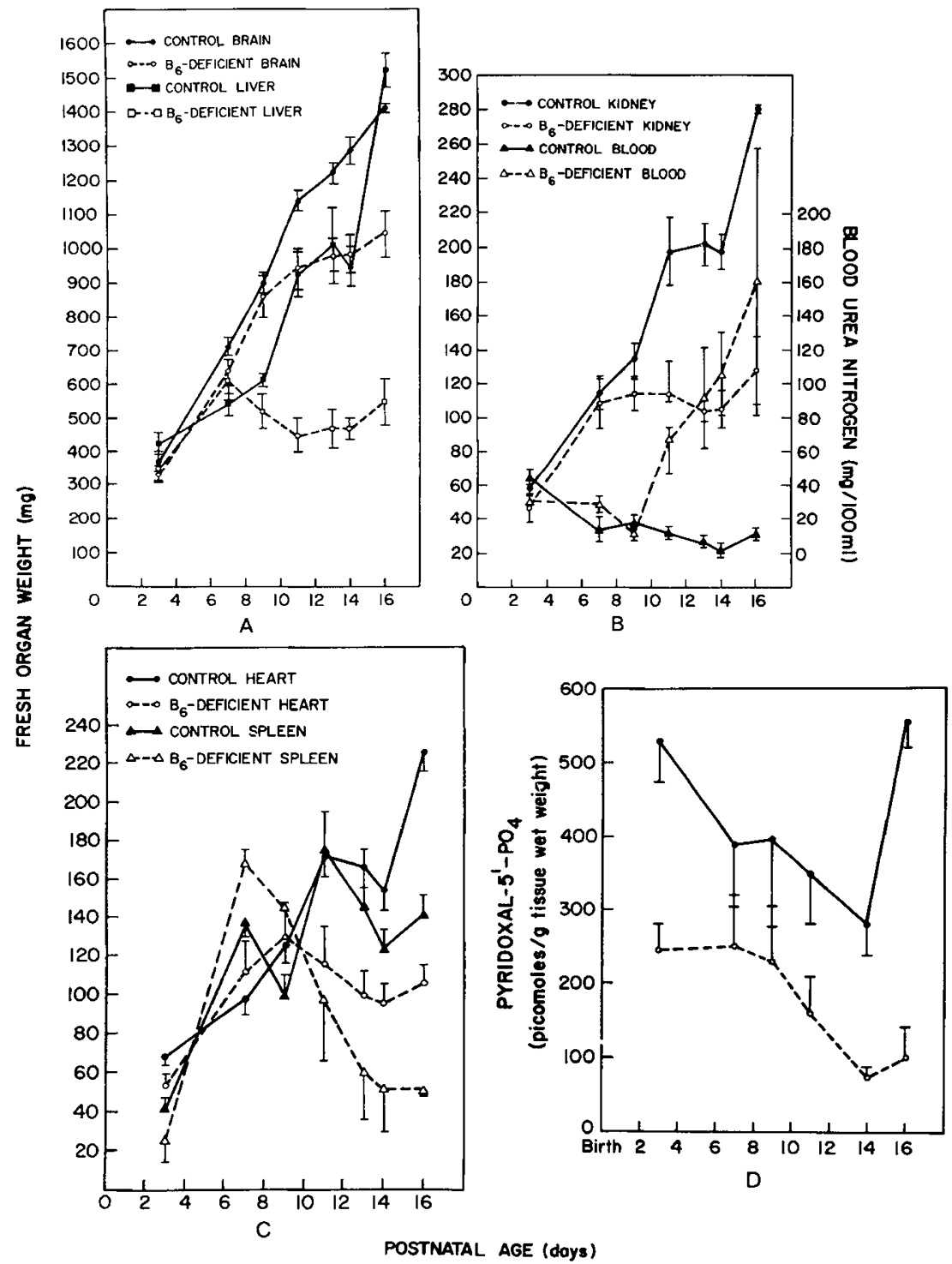

Fig. 1. Effect of vitamin $\mathrm{B}_{6}$ deficiency on the weights of brain $(A)$, liver $(A)$, kidney $(B)$, heart $(C)$, and spleen $(C)$; the level of blood urea nitrogen (lB); and the concentration of pyridoxal-5'-phosphate $\left(D:-\longrightarrow\right.$, control; $\mathrm{O}_{-} \ldots$ nutritionally deprived) during development. Each point represents the average of eight values \pm SD. 


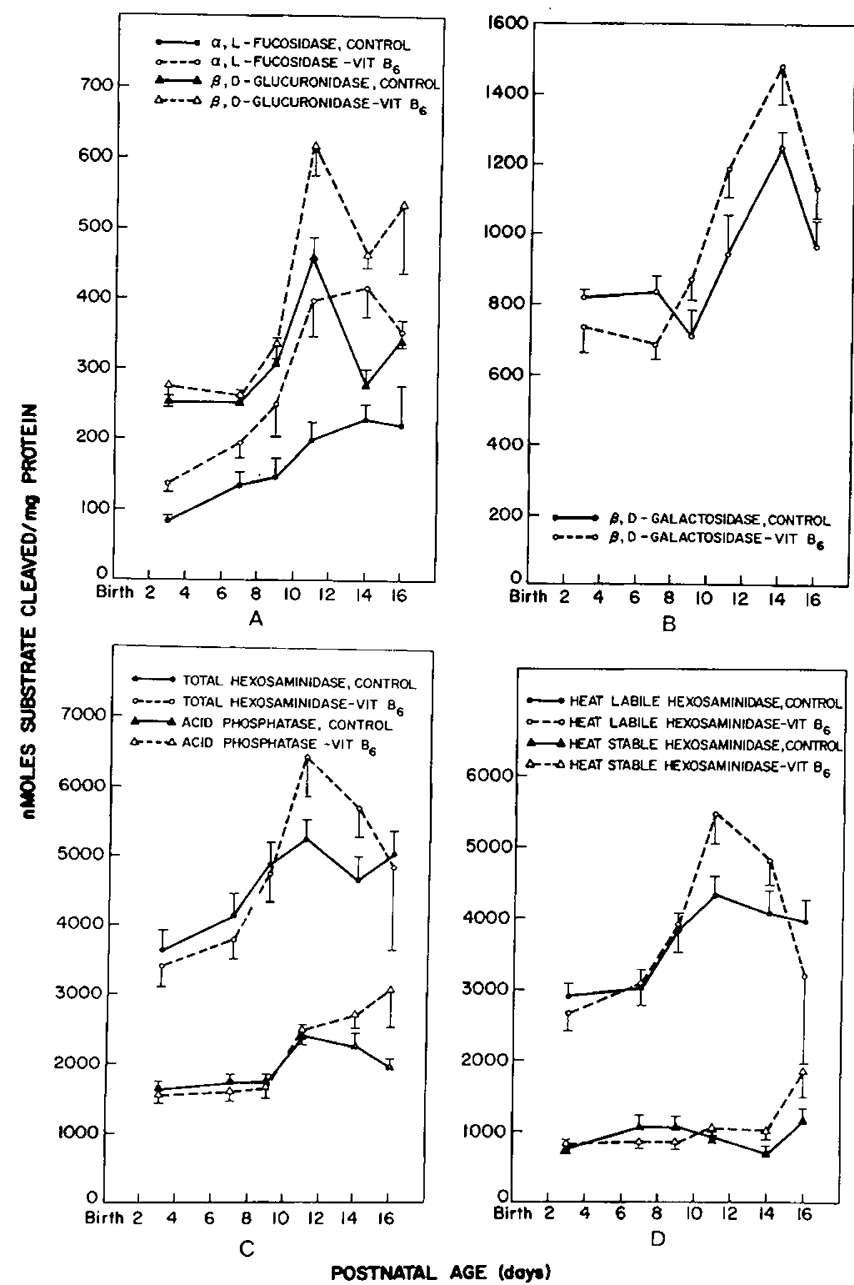

Fig. 2. Effect of vitamin $B_{6}$ deficiency on the renal activities of $\alpha$-L-fucosidase $(A), \beta$-D-glucuronidase $(A), \beta$-D-galactosidase $(B)$, total hexosaminidase $(C)$, acid phosphatase $(C)$ and hexosaminidases $\mathrm{A}$ and $\mathrm{B}$ $(D)$ during development. Each point represents the average of eight values $\pm \mathrm{SD}$.

indivdual homogenates of kidney used in determining the activities of lysosomal acid hydrolases.

\section{RESULTS}

\section{ORGAN WEIGHTS}

Growth of organs in control and $B_{6}$-deficient groups was similar between 3 and 9 days of age (Fig. 1). Differences in the organ weight in $\mathrm{B}_{6}$-deficient animals became apparent after the 9 th postnatal day. The kidney (Fig. $1 B$ ) spleen (Fig. $1 C$ ) heart (Fig. $1 C$ ) and liver (Fig. 1 $A$ ) from deficient young either plateaued and failed to gain weight or lost weight resulting in mean weights of $35-50 \%$ of controls by 16 postnatal days. The brain of $B_{6}$-deficient young continued to gain weight from 9 days onward but at a more reduced rate than normal (Fig. 1 $A$ ). By the 16 th postnatal day, only a $25 \%$ decrease in brain weight was observed in deficient animals. The levels of protein, expressed as milligrams per gram of tissue wet weight, in kidney and brain of deprived animals was calculated and found to be similar to normal controls at all ages.

\section{BLOOD UREA NITROGEN (BUN)}

BUN remained normal in $\mathrm{B}_{6}$-deficient young during the 3-9-day period but had increased to $160 \mathrm{mg} / 100 \mathrm{ml}$ by the 16 th postnatal day (Fig. 1B).

\section{CONTENT OF PYRIDOXAL-5'-PHOSPHATE IN KIDNEY}

Pyridoxal-5'-phosphate content in normal kidney decreased during the time period from 3-14 postnatal days but returned to the 3-day level by the 16th day (Fig. $1 D$ ). The level of cofactor in kidney of experimental animals was below that of controls at all time points studied (Fig. 1D). During the period between 3 and 9 days the concentration remained unchanged but subsequently decreased coinciding with a failure of kidney to gain weight and elevation of BUN (Fig. $1 B$ ).

\section{LYSOSOMAL ACID HYDROLASE ACTIVITY}

Kidney. Specific activities of $\alpha$-L-fucosidase, $\beta$ - $\mathrm{D}$-glucuronidase, $\beta$-D-galactosidase, acid phosphatase, hexosaminidases A and $\mathrm{B}$, and $\alpha$-D-mannosidase increased during the time period between 3 and 16 postnatal days (Figs. 2 and 3). Glucuronidase was the only enzyme studied showing a biphasic curve with a peak in activity at 11 days and a second one developing starting at 14 days (Fig. $2 A$ ). Similar patterns of enzyme activity were observed in $B_{6}$-deficient kidney. The specific activities of the lysosomal hydrolases in homogenates of kidney from deficient animals were unchanged between 3 and 9 days of age. $\alpha$-L-Fucosidase activity (Fig. 2A) was already moderately increased $(37 \%)$ in deficient kidney at 3 postnatal days, whereas a small decrease in $\beta$-D-galactosidase activity (Fig. $2 B$ ) was observed. The activity of $\alpha$-Lfucosidase remained elevated throughout the time course, reaching a maximum of $400 \mathrm{nmol}$ of substrate hydrolyzed $/ \mathrm{mg}$ protein between 11 and 14 days, a value nearly $50 \%$ greater than that of the control animals. $\beta$-D-Glucuronidase (Fig. $2 A$ ), $\beta$-D-galactosidase (Fig. $2 B$ ), acid phosphatase (Fig. $2 C$ ), and hexosaminidases $A$ and $\mathrm{B}$ (Fig. $2 D$ ) in vitamin $\mathrm{B}_{6}$-deficient young all showed moderate $(25-30 \%)$ increases in activities, after 9 days at one or more time points. The difference in hexosaminidase $\mathrm{A}$ activity disappeared by the 16th postnatal day, although the level of hexosaminidase B remained elevated. The activity of $\alpha$-D-mannosidase (Fig. 3) in deficient kidney was unchanged during the time period studied

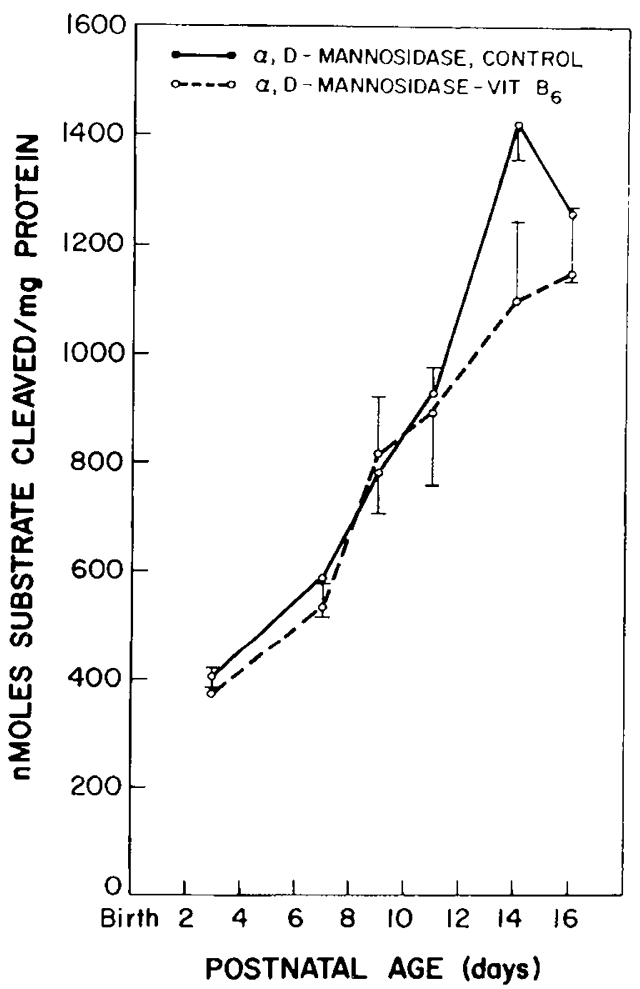

Fig. 3. Effect of vitamin $B_{6}$ deficiency on the renal activity of $\alpha$-D-mannosidase during development. Each point represents the average of eight values $\pm \mathrm{SD}$. 


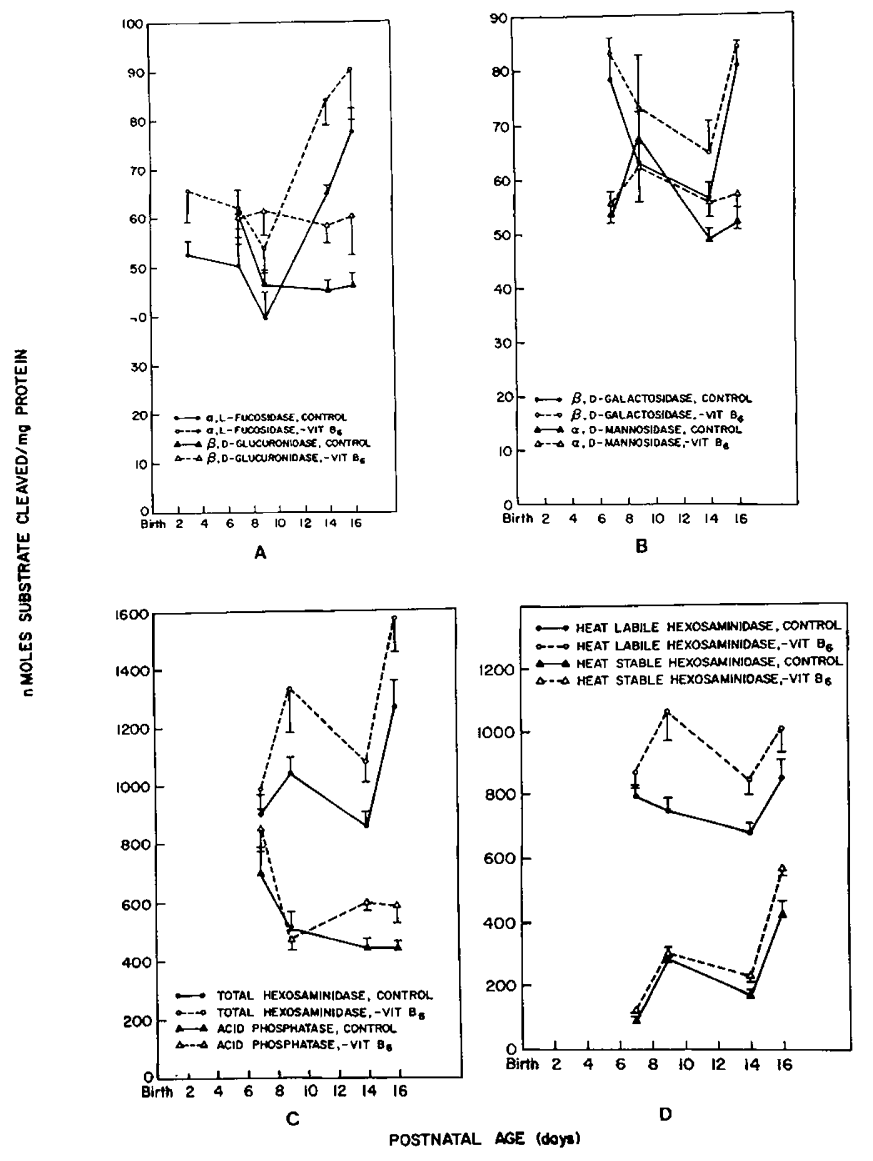

Fig. 4. Effect of vitamin $B_{6}$ deficiency on the cerebral activities of $\alpha$-L-fucosidase $(A), \beta$-D-glucuronidase $(A), \beta$-D-galactosidase $(B), \alpha$-Dmannosidase $(B)$, total hexosaminidase $(C)$, acid phosphatase $(C)_{4}$ and hexosaminidases $\mathrm{A}$ and $\mathrm{B}(D)$ during development. Each point represents the average of eight values \pm SD.

except for a small decrease at 14 days of age. There was no apparent direct correlation between the level of pyridoxal- 5 '-phosphate in kidney and the activity of the lysosomal hydrolases.

Brain. Developmental patterns of hydrolase activity in control brain (Fig. 4) were different and more complex than those observed in control kidney. $\alpha$-L-Fucosidase (Fig. $4 A$ ), $\beta$-D-galactosidase (Fig. $4 B$ ), and hexosaminidases A and B (Fig. $4 D$ ) were still increasing by 16 postnatal days, the latest point examined, whereas the activities of $\beta$-D-glucuronidase (Fig. $4 A$ ), $\alpha$-D-mannosidase (Fig. $4 B$ ), and acid phosphatase (Fig. $4 C$ ) had plateaued. The patterns of activity in $\mathrm{B}_{6}$-deficient brain were similar to controls but, as in deficient kidney, the hydrolase activities were elevated. $\alpha$-L-Fucosidase activity (Fig. $4 A$ ) was increased somewhat throughout the time course, but changes in $\beta$-D-glucuronidase (Fig. 4A), $\beta$-D-galactosidase (Fig. 4B), $\alpha$-D-mannosidase (Fig. $4 B$ ), acid phosphatase (Fig. $4 C$ ), and hexosaminidase $A$ and B (Fig. 4D) did not occur until after 7 postnatal days. The greatest changes in enzyme activities in deficient brain were observed during the time period from 9 to 14 days of age, after which the differences in $\beta$-D-galactosidase, $\alpha$-D-mannosidase, and hexosaminidase A disappeared.

\section{DISCUSSION}

The weights of kidney (Fig. $1 B$ ), brain (Fig. $1 A$ ), liver (Fig. $1 A$ ), heart (Fig. $1 C$ ) and spleen (Fig. $1 C$ ) from normal control subjects increased during the period between 3 and 16 postnatal days. Similar patterns of growth have already been reported $(24,26)$. These organs from vitamin $\mathrm{B}_{6}$-deficient young, in concert, ceased to continue to gain weight on or about the 10th postnatal day coinciding with a failure in the whole body to grow (3).
The levels of pyridoxal-5'-phosphate in normal control kidney during postnatal development have not been reported previously. We observed a decrease in concentration from 3 to 14 postnatal days followed by return to the 3-day level by 16 postnatal days (Fig. $I D$ ). A marked absence of pyridoxal-5'-phosphate in kidneys from vitamin deprived young before 10 days did not appear to have an effect on renal growth. Blood urea nitrogen in $B_{6}$-deficient young increased markedly during the period between 10 and 16 days, coinciding with a failure in the kidney to grow. The uremia observed in deficient animals may have been caused by an increased oxidation of amino acids for energy production (5) or may have been the consequence of structural alterations of the kidney known to occur in vitamin $B_{6}$ deficiency (3). The content of pyridoxal- $5^{\prime}$-phosphate in liver and brain was not measured in this study. It had been shown to be lower than normal on the 10th and 12 th postnatal days (3). The degree of depletion in both organs on day 10 , brain $20 \%$ of normal and liver $50 \%$ of normal, would suggest a deficiency even at an earlier age.

The activities of the lysosomal hydrolases measured in control kidney increased during the period between 3 and 16 postnatal days (Figs. 2, 3). Determinations were not made beyond the 16th day. Similar normal developmental profiles, during this period, have been described for $\beta$-D-galactosidase and $\beta$-D-glucuronidase by Koldovsky (10) and Raychaudhuri and Desai (20). There appears to be a descrepancy as to what course the activities of these enzymes follow after weaning. Raychaudhuri and Desai (20) reported that $\beta$-D-galactosidase is not changed between 20 and 30 days of age and that $\beta$-D-glucuronidase is still increasing in activity during this time period. On the other hand, Koldovsky (10) found that the activities of both enzymes decreased between the 20th and 30 th postnatal days.

The developmental profile for renal acid phosphatase (Fig. 2C) was comparable with that reported by Raychaudhuri and Desai (20). Patterns of activities in developing kidney for $\alpha$-L-fucosidase, hexosaminidase, and $\alpha$-D-mannoside have not been reported previously.

There have also been conflicting reports on the patterns of lysosomal acid hydrolase activities in developing brain. Traurig et al. (25) reported decreases in both $\beta$-D-glucuronidase and acid phosphatase postnatally, whereas Raychaudhuri and Desai (20) reported a similar decrease in acid phosphatase but an increase in $\beta$-D-glucuronidase. Our data for these hydrolases (Fig. $4 A, C$ ), as well as that of hexosaminidase (Fig. $4 D$ ), are comparable with those of Traurig et al. (25). Both $\alpha$-D-mannosidase and $\alpha$-L-fucosidase have been shown to decrease with age in brain (19). We also found the activity of $\alpha$-D-mannosidase (Fig. $4 B$ ) to decrease postnatally but observed a marked increase in $\alpha$-L-fucosidase activity (Fig. $4 A$ ). The developmental profile of $\beta$-D-galactosidase (Fig. $4 B$ ) was different from that already described (20).

There is no ready explanation for the conflicting reports on the developmental profiles of the lysosomal acid hydrolases in kidney and brain. In all the studies that have been compared, activities were measured in whole homogenates. Variations in the assay systems including $\mathrm{pH}$, buffer system, artificial substrate used (i.e., $P$-nitrophenyl vs. 4-methylumbelliferyl derivatives), and the use of detergents, which may have resulted in differences in specific activities, should not have influenced the developmental patterns and profiles of the enzymes.

The reduction of vitamin $B_{6}$ content in kidney and brain had little effect on the developmental profiles of the acid hydrolases examined but did result in changes in the specific activities of enzymes when compared with controls at one or more age points. Except for $\alpha$-D-mannosidase, which showed a decrease in activity at 14 days, the activities of most enzymes were elevated in kidney by the 11th postnatal day. In brain from deficient animals, increases in acid hydrolases were also observed, however, the changes were not as dramatic as those found in deficient kidney. $\alpha$-L-Fucosidase appeared to be most sensitive to an absence in vitamin $B_{6}$ since its activity in both kidney and brain was already elevated at 3 days of age, when the levels of activities of other hydrolases were normal. 
Specific activities of several lysosomal hydrolases have also been shown to increase markedly in the liver (1) and intestine (2) of fasted rats, coinciding with decreases in the weights of these organs. On resumption of feeding, enzyme activities rapidly returned to normal levels.

Because of the close relationship between increased enzyme activity and decreased organ weight, it was suggested that lysosomal acid hydrolases play important roles in conditions under which dietary nutrients are not available. They function to catalyze the breakdown of cellular organelles and constituents, the products of digestion being used for energy production and for precursors for essential tissue reconstruction so that the animal can survive.

Increased lysosomal hydrolase activity, observed in vitamin $\mathrm{B}_{6}$-deficient kidney and brain when the weights of these organs were lower than normal, may reflect their inadequate food intake resulting from an inability of deprived young to suckle normally. However, at 3 postnatal days, when both the weights of kidney and brain were normal, there was already a marked elevation in $\alpha$-L-fucosidase activity in these organs, suggesting that this increase in enzyme activity may be a specific effect of vitamin $B_{6}$ deficiency.

Whether the changes in lysosomal hydrolase activity were the consequence of increased synthesis of enzymic proteins, decreased degradation of enzymic proteins, increased degradation of nonenzymic proteins, or a combination of these could not be determined.

Studies directed toward examining renal morphology in vitamin $\mathrm{B}_{6}$ deficiency employing the electron microscope are underway currently in this laboratory.

\section{SUMMARY}

The rate of weight gain of kidney, brain, liver, spleen, and heart from developing animals deprived of vitamin $B_{6}$ during pregnancy or postnatally was less than that of controls. Specific activities of renal and cerebral lysosomal hydrolases were increased, especially the activity of $\alpha$-L-fucosidase which was already elevated in these organs before any weight changes.

\section{REFERENCES AND NOTES}

1. Desai, 1. D.: Regulation of lysosomal enzymes. I. Adaptive changes in enzyme activities during starvation and refeeding. Can. J. Biochem., 47: 785 (1969).

2. Desai, I. D.: Regulation of lysosomal enzymes. II. Reversible adaption of intestinal acid hydrolases during starvation and realimentation. Can. J. Biochem., 49: 170 (1971).

3. DiPaolo, R. V., Caviness, V. S., and Kanfer, J. N.: Delayed maturation of the renal cortex in the vitamin $B_{6}$-deficient newborn rat. Pediat. Res., 8: 546 (1974).

4. French, S. W.: Effect of chronic ethanol ingestion on liver enzyme changes induced by thiamine, riboflavin, pyridoxine or choline deficiency. J. Nutr., 88: 291 (1966).
5. Hawkins, W. W., MacFarland, M. L., and McHenry, E. W.: Nitrogen metabolism in pyridoxine insufficiency. J. Biol. Chem., 166: 223 (1946).

6. Hultberg, B., and Ockerman, P. A.: Artificial substrates in the assay of acid glycosidases. Clin. Chim. Acta, 39: 49 (1972).

7. Itzhaki, R. F., and Gill, D. M.: A microbiuret method for estimating proteins. Anal. Biochem., 9: 401 (1964).

8. Kanfer, J. N., and Spielvogel, C.: Hexosaminidase activity of cultured human skin fibroblasts. Biochim. Biophys. Acta, 193: 203 (1973).

9. Kanfer, J. N., Spielvogel, C., and Milunsky, A.: The effect of p-chloromercuriophenylsulfonate on skin fibroblast acid hydrolases. Life Sci., 11: 191 (1972).

10. Koldovsky, $O$.: Developmental changes of $\beta$-galactosidase and $\beta$-glucuronidase in the rat liver and kidney. Arch. Biochem. Biophys., 142, 378 (1971).

11. Kurtz, D. J., Levy, H., and Kanfer, J. N.: Cerebral lipids and amino acids in the vitamin $\mathrm{B}_{6}$-deficient suckling rat. J. Nutr., 102: 291 (1972).

12. Leaback, D. H., and Walker, P. G.: Studies on glucosaminidase. 4. The fluorimetric assay of $n$-acetyl- $\beta$-glucosaminidase. Biochem, J., 78: 151 (1961).

13. Levvy, G. A., and McAllan, A.: Mammalian fucosidases. 1. The synthesis of substrates and inhibitors. Biochem. J., 80: 433 (1961).

14. Levvy, G. A., and McAllan, A.: Mammalian fucosidases. 2. $\alpha$-L-Fucosidase Biochem. J., 80: 435 (196!).

15. Maruyama, H., and Coursin, D. B.: Enzymatic assay of pyridoxal phosphate using tyrosine apodecarboxylase and tyrosine-1-C ${ }^{14}$. Anal. Biochem., 26: 420 (1968).

16. Mead, J. A. R., Smith, J. N., and Williams, R. T.: Studies on detoxication: The biosynthesis of the glucuronides of umbelliferone and 4-methylumbelliferone and their use in fluorimetric determination of $\beta$-glucuronidase. Biochem. J., 61 : 569 (1955).

17. Milunsky, A., Spielvogel, C., and Kanfer, J. N.: Lysosomal enzyme variations in cultured normal skin fibroblasts. Life Sci., 11: 1101 (1972).

18. O'Brien, J. S., Okada, S., Chen, A., and Fillerup, D. L.: Tay-Sachs disease: Detection of heterozygotes and homozygotes by serum hexosaminidase assay. N. Engl. J. Med. 283: 15 (1970).

19. Quarles, R. H., and Brady, R. O.: Sialoglycoproteins and several glycosidases in developing rat brain. J. Neurochem., 17: 801 (1970).

20. Raychaudhuri, C., and Desai, I. D.: Regulation of lysosomal enzymes. IV. Changes in enzyme activities of liver, kidney, and brain during development. Int. J. Biochem., 3: 309 (1972).

21. Robinson, D.: Fluorimetric determination of glycosidases in the locust (Locusta migratoria) and other insects. Comp. Biochem. Physiol., 12: 95 (1964).

22. Robinson, D., Price, R. G., and Dance, N.: Separation and properties of $\beta$-galactosidase, $\beta$-glucosidase, $\beta$-glucuronidase, and $n$-acetyl- $\beta$-glucosaminidase from rat kidney. Biochem. J., 102: 525 (1967).

23. Robinson, D., and Willcox, P.: 4-Methylumbelliferyl phosphate as a substrate for lysosomal acid phosphatase. Biochim. Biophys. Acta, 19l: 183 (1969).

24. Solomon, S., and Bengele, H. H.: Organ rates and organ weights of rats. Biol. Neonate, 22: 222 (1973).

25. Traurig, H. H., Clendenon, N. R., Swenberg, J. A., and Allen, N.: Lysosomal acid hydrolases in neonatal rat brain. J. Neurobiol., 4: 105 (1973).

26. Zeeman, F. J.: Effect of protein deficiency during gestation on postnatal cellular development in the young rat. J. Nutr., 100: 530 (1970).

27. Wilmington, Mass.

28. Fisher Scientific Co.,

29. New England Kennel Co.,

30. Nutritional Biochemicals, Cleveland, Ohio.

31. Dow Chemical, Indianapolis, Ind.

32. Heat Systems-Ultrasonics, Inc., New York, N. Y.

33. The authors thank Marjorie L. Scott for performing the blood urea nitrogen analyses.

34. This work supported by United States Public Health Service Grants HD 05515, HD 04147, and NS 10330 and Maternal and Child Health Project 906.

35. Requests for reprints should be addressed to: J. N. Kanfer, Ph.D., E. K. Shriver Center, 200 Trapelo Rd., Waltham, Mass. 02154 (USA).

36. Accepted for publication April 22, 1975. 